\title{
Resolving the dark matter of ABCA4 for 1054 Stargardt disease probands through integrated genomics and transcriptomics
}

\author{
A full list of authors and affiliations appears at the end of the paper.
}

\begin{abstract}
Purpose: Missing heritability in human diseases represents a major challenge, and this is particularly true for $A B C A 4$-associated Stargardt disease (STGD1). We aimed to elucidate the genomic and transcriptomic variation in 1054 unsolved STGD and STGD-like probands.
\end{abstract}

Methods: Sequencing of the complete 128-kb $A B C A 4$ gene was performed using single-molecule molecular inversion probes (smMIPs), based on a semiautomated and cost-effective method. Structural variants (SVs) were identified using relative read coverage analyses and putative splice defects were studied using in vitro assays.

Results: In 448 biallelic probands 14 known and 13 novel deepintronic variants were found, resulting in pseudoexon (PE) insertions or exon elongations in 105 alleles. Intriguingly, intron 13 variants c. $1938-621 \mathrm{G}>\mathrm{A}$ and c. $1938-514 \mathrm{G}>\mathrm{A}$ resulted in dual $\mathrm{PE}$ insertions consisting of the same upstream, but different downstream PEs. The intron 44 variant c.6148-84A $>$ T resulted in two PE insertions and flanking exon deletions. Eleven distinct large deletions were found, two of which contained small inverted segments. Uniparental isodisomy of chromosome 1 was identified in one proband.

Conclusion: Deep sequencing of $A B C A 4$ and midigene-based splice assays allowed the identification of SVs and causal deepintronic variants in $25 \%$ of biallelic STGD1 cases, which represents a model study that can be applied to other inherited diseases.

Genetics in Medicine (2020) 22:1235-1246; https://doi.org/10.1038/s41436020-0787-4

Keywords: $A B C A 4$; Stargardt disease; smMIPs; deep-intronic variants; structural variants

\section{INTRODUCTION}

High-throughput genome sequencing has made a huge impact in biology and is considered the most powerful genetic test to elucidate inherited human diseases. ${ }^{1}$ It allows the unbiased detection of a wide spectrum of genetic variants including coding and noncoding single-nucleotide variants (SNVs), as well as structural variants (SVs). However, sequencing and data storage costs as well as the possibility of secondary genetic findings hamper the use of genome sequencing.

Based on the advantages and limitations mentioned above, genome sequencing is not the best method to perform sequence analysis of one or a few genes that are associated with a clinically distinct condition. This is illustrated by autosomal recessive Stargardt disease (STGD1), which is caused by variants in the $A B C A 4$ gene. STGD1 is the most frequently inherited macular dystrophy with an estimated prevalence of $1 / 10,000 .^{2}$ Thus far, 1180 unique $A B C A 4$ variants have been reported in 8777 alleles of 6684 cases (www.lovd.nl/ABCA4). ${ }^{3}$ A large proportion of the variants affect noncanonical splice site (NCSS) sequences, with variable effects on messenger RNA (mRNA) processing, ${ }^{4-6}$ and several deep-intronic (DI) variants have been identified. ${ }^{5,7-13}$ Most of these DI variants strengthen cryptic splice sites resulting in the insertion of pseudoexons (PEs) in the mature $A B C A 4$ mRNA. SVs seem to be rare in $A B C A 4,7,10,12,14$ although systematic copy-number variant $(\mathrm{CNV})$ analyses have not been performed in most STGD1 cases.

Due to the relatively large size of the $A B C A 4$ gene (50 exons; $128,313 \mathrm{bp}$ ), variant screening initially was restricted to the scanning of the exons and flanking splice sites with poor sensitivity, leaving $50-70 \%$ of STGD1 probands genetically unsolved. ${ }^{14-17}$ Recently, sequence analysis of the entire $128-\mathrm{kb}$ gene was performed using next-generation sequencing platforms using Raindance microdroplet polymerase chain reaction (PCR) target enrichment or Illumina TruSeq Custom Amplicon target enrichment, ${ }^{10}$ HaloPlex-based sequence enrichment, ${ }^{7,9}$ or genome sequencing. ${ }^{1,9}$

Identification of two pathogenic alleles is important to confirm the clinical diagnosis because several promising clinical trials are underway based on RNA modulation with antisense oligonucleotides, ${ }^{7,9,18}$ drug based therapies, ${ }^{19}$ as well as gene augmentation ${ }^{20}$ and stem cell therapies. ${ }^{21}$ STGD1

Correspondence: Frans P. M. Cremers (frans.cremers@radboudumc.nl)

These senior authors contributed equally: Claire-Marie Dhaenens, Frans P. M. Cremers 
cases will only be eligible for these therapies if both causal alleles are known. In addition, recent studies identified alleles carrying a coding variant in cis with a DI variant, and only these combinations represented fully penetrant alleles, ${ }^{7,9}$ pointing toward the importance of analyzing noncoding regions in the STGD1 cases.

Recently, we reported on the use of 483 single-molecule molecular inversion probes (smMIPs) to sequence the 50 exons and 12 intronic regions carrying 11 pathogenic DI variants of 412 genetically unsolved STGD1 cases. ${ }^{5}$ In this study, we aimed to design a semiautomated, high-throughput, cost-effective, and comprehensive sequence analysis of the entire $A B C A 4$ gene, which could serve as a model study to investigate human inherited diseases due to variants in one or a few genes. Using 3866 smMIPs we sequenced 1054 genetically unsolved STGD or STGD-like probands and 138 biallelic controls carrying known $A B C A 4$ variants. Novel NCSS and DI variants were tested in vitro for splice defects. Additionally, a very high and reproducible read coverage allowed us to perform CNV analysis.

\section{Samples}

\section{MATERIALS AND METHODS}

Twenty-one international and four national centers ascertained 1054 genetically unsolved probands in whom STGD was part of the differential diagnosis as determined by the local ophthalmologists specializing in inherited retinal diseases. Since $A B C A 4$ disease is known for its clinical heterogeneity, a spectrum of (overlapping) $A B C A 4$-associated phenotypes were part of this study, as well as a STGD1 phenocopy: central areolar choroidal dystrophy (CACD). The clinical findings specific to a certain clinical diagnosis and the main phenotypic characteristics used in the differential diagnosis are described in Table S1. Also, 19 cases with a clinical diagnosis of macular dystrophy without further specification were included.

Among 1054 cases 833 probands were previously screened by employing different screening methods, i.e., exome sequencing, targeted gene panel sequencing including all $A B C A 4$ coding regions, and Sanger sequencing of all coding $A B C A 4$ exons. Details are provided in Table S2.

We discerned two patient groups. The first patient group consisted of 993 genetically unsolved probands who carried one $(n=345)$ or no $(n=648) A B C A 4$ allele. For two subjects, DNA was not available and both parents of the probands were studied, assuming autosomal recessive inheritance. The second patient group consisted of 61 "partially solved" probands, carrying the c.5603A $>\mathrm{T}$ (p.Asn1868Ile) variant in trans with other alleles. This last group was also investigated as it was suspected that there could be unidentified DI variants in cis with c.5603A $>\mathrm{T}$, as the penetrance of c. $5603 \mathrm{~A}>\mathrm{T}$, when in trans with a severe $A B C A 4$ variant, was $\sim 5 \%$ in the population. ${ }^{22,23}$

This study was approved by the Medical Ethical Committee 2010-359 (Protocol nr. 2009-32; NL nr. 34152.078.10) and the Commissie Mensgebonden Onderzoek Arnhem-Nijmegen
(Dossier no. 2015-1543; dossier code sRP4h). All samples were collected according to the tenets of the Declaration of Helsinki and written informed consent was obtained for all patients participating in the study.

\section{smMIPs design and $A B C A 4$ sequence analysis}

Detailed information on the smMIPs-based $A B C A 4$ sequencing, selection of candidate splice variants, and inclusion criteria is provided in the Supplementary Materials and Methods.

\section{Midigene-based splice assay}

The effect of nine NCSS variants and 58 DI variants was assessed by midigene-based splicing assays employing 23 wild-type (WT) BA clones previously described ${ }^{4}$ and the newly designed BA32, BA33, BA34, and BA35. WT and mutant constructs were transfected in HEK293T cells and the extracted total RNA was subjected to reverse transcription (RT)-PCR as described previously. ${ }^{4}$ Details are provided in Supplementary Materials and Methods.

\section{Identification of CNVs and assessment of the underlying mechanism}

An Excel-based script was employed to detect CNVs using smMIP read number. Microhomology at the breakpoints was assessed using ClustalW, breakpoint regions were analyzed for non-B motifs by tool (nBMST and QGRS Mapper) (for details see Supplementary Materials and Methods).

\section{Semiquantification of RT-PCR products}

To quantify the ratios between correct and aberrant RT-PCR products, densitometric analysis was performed using ImageJ software.

\section{Uniparental disomy detection}

To test the presence of uniparental disomy (UPD), haplotype analysis was performed in one STGD1 case (DNA14-33085) using exome sequencing data.

\section{RESULTS}

\section{smMIPs performance and $A B C A 4$ sequencing}

A pilot sequencing study was conducted using 15 STGD1 samples and five DNA samples of control individuals, revealing all 34 previously identified variants (Table S3). The average number of reads for the 20 DNA samples ranged from 10 to 152,500 per smMIP, with an overall average coverage of $933 \times$ for each smMIP.

In total 1192 DNA samples were analyzed for variants in $A B C A 4$ using six NextSeq500 runs. The average number of reads of the 3866 smMIPs was $377 \times$. As most nucleotide positions are targeted with two smMIPs, the effective average coverage was $\sim 700 \times$. To determine the coverage of $A B C A 4$ in more detail, we calculated the average coverage of each nucleotide position for runs 1 to 5 combined (Table S4). To visualize the results, nucleotide positions that were not covered or poorly covered ( $\leq 10$ reads), moderately covered 
a

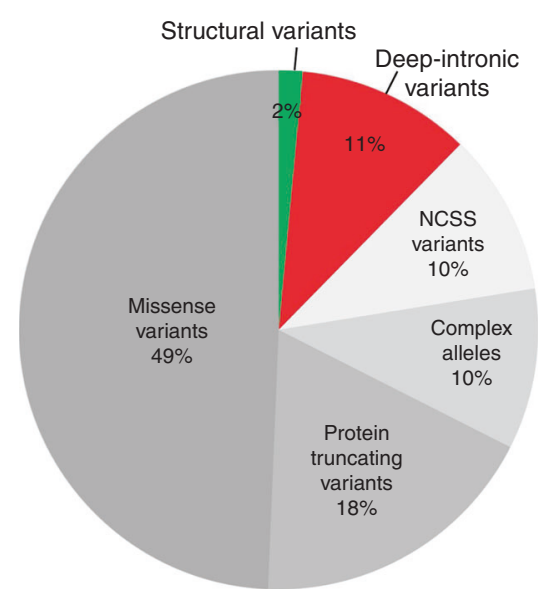

b

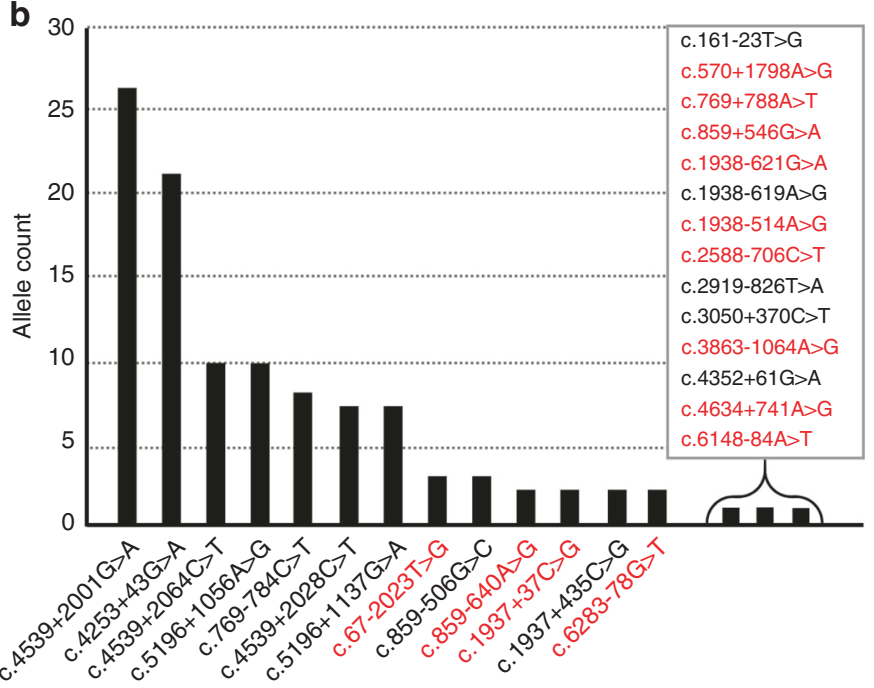

Fig. 1 Distribution of different types of alleles and deep-intronic variants in ABCA4. a The contribution of each type of variant or allele in biallelic and monoallelic cases except those carrying c.5603A>T is represented. Protein truncating variants comprise nonsense, frameshift, and canonical splice site variants. The $10 \%$ complex alleles only consist of combinations of missense variants, the most frequent of which were c.[1622T>C;3113C $>T](n=30 ; 27 \%$ of all complex alleles) and c.[4469G>A;5603A>T] ( $n=27 ; 25 \%$ of all complex alleles). They do not include the complex alleles that contain noncanonical splice site (NCSS) variants, deep-intronic variants, or protein truncating variants, when present in cis with other variants. If these would have been included, $16 \%$ of the alleles would consist of complex alleles. b Deep-intronic variant allele count in this study. Novel deep-intronic variants are highlighted in red. One hundred seventeen causal deep-intronic variants were identified. The deep-intronic variants c.4539+2001G $>$ A $(n=26)$ and c.4253 $+43 G>A(n=21)$ were found most frequently. Most of the novel deep-intronic variants were found in single STGD1 probands.

(11-49 reads), or well covered ( $\geq 50$ reads) are depicted in Fig. S1. From the $128,366 \mathrm{nt}$ of $A B C A 4,1980 \mathrm{nt}(1.5 \%)$ were not or poorly covered, $1410 \mathrm{nt}(1.1 \%)$ were moderately covered, and 124,976 nt (97.4\%) were well covered. Although $A B C A 4$ introns carry several repetitive elements (Fig. S1), they only had a small effect on smMIPs design. Several larger repeats are present in up- and downstream regions of $A B C A 4$, which resulted in the absence-or poor performance-of smMIPs. Sequencing of 1192 samples yielded a total of 7756 unique $A B C A 4$ variants that are listed in Table S5.

\section{Sensitivity and specificity of the smMIPs-based sequencing} To assess the sensitivity of the new smMIPs sequencing platform, we tested 123 previously genotyped samples ${ }^{5,9}$ in three series (runs 2, 3, and 6) (Table S6) as well as 15 control DNA samples carrying 13 different SVs spread throughout the $A B C A 4$ gene (run 6) (Table S7). All previously known SNVs $(n=300)$ and 13 SVs could be identified, yielding a sensitivity of $100 \%$. Six additional variants were found due to low coverage in the previous studies, and three variants had not been annotated correctly previously.

\section{$A B C A 4$ gene sequencing and identification of variants}

$A B C A 4$ sequencing was performed for 1054 genetically unsolved STGD and STGD-like patients. This revealed 323 unique (likely) pathogenic SNVs and 11 SVs in 1144 alleles. Sixty-four of 323 SNVs (26\%) and all 11 SVs were novel (Table S8). Detailed in silico analysis of novel SNVs is provided in Table S9. Thirteen percent of these alleles were represented by DI variants and SVs and another $10 \%$ accounted for NCSS variants (Fig. 1a). All variants and the respective cases were uploaded into the $A B C A 4$ variant and STGD1 cases database LOVD at www.lovd.nl/ABCA4.

Two (likely) pathogenic variants were found in 323 probands, three probands carried p.Asn1868Ile in a homozygous manner, and one (likely) causal variant in trans with $\mathrm{p}$. Asn1868Ile was found in 125 probands. Only one (likely) causal variant was identified in 174 probands. Additionally, in 65 probands, the p.Asn1868Ile variant was the only identified variant (Table S10). No (likely) causal variants were found in 364 cases.

Among the SNVs, the most common causal alleles were c. $5603 \mathrm{~A}>\mathrm{T} \quad(n=134), \quad$ c. $5882 \mathrm{G}>\mathrm{A} \quad(n=84), \quad$ c. $[5461-$ $10 \mathrm{~T}>\mathrm{C} ; 5603 \mathrm{~A}>\mathrm{T}] \quad(n=44), \quad$ c. $[1622 \mathrm{~T}>\mathrm{C} ; 3113 \mathrm{C}>\mathrm{T}] \quad(n=30)$, c. $[4469 \mathrm{G}>\mathrm{A} ; 5603 \mathrm{~A}>\mathrm{T}] \quad(n=27)$, c. $4539+2001 \mathrm{G}>\mathrm{A} \quad(n=26)$, c. $6079 \mathrm{C}>\mathrm{T}(n=23)$, and c.4253+43G $>\mathrm{A}(n=21)$ (Table S8). To visualize the relative frequency of causal STGD1-causing alleles, we excluded 65 heterozygous c. $5603 \mathrm{~A}>\mathrm{T}$ alleles that were found as the only $A B C A 4$ allele in these cases, as they were most likely present because of its high allele frequency (0.06) in the general population (Fig. S2). ${ }^{23,24}$

\section{Splice defects due to noncanonical splice site variants}

The effect on splicing of nine NCSS variants was tested in nine wild-type splice constructs previously described ${ }^{4}$ (Fig. S3). All of the nine tested novel NCSS variants showed a splice defect when tested in HEK293T cells. Severity was assigned according to the percentage of remaining WT mRNA, as described previously. ${ }^{4}$ Five NCSS variants were deemed severe as they showed $\leq 30$ of WT mRNA, three were 
considered to have a moderate effect with WT RNA present between $>30$ and $\leq 70 \%$ correct RNA and only one was mild as it showed $>70 \%$ of WT RNA (Table S11, Fig. S4).

\section{Deep-intronic variants identification and functional characterization}

Based on the defined selection criteria, 58 DI variants were selected for splice assays. To test their effects, $27 \mathrm{WT}$ midigene splice constructs were employed, 23 of which were described previously, ${ }^{4}$ and four of which were new (Fig. S3). Thirteen of 58 tested DI variants showed a splice defect upon RT-PCR and Sanger validation (Figs. 2 and 3). For the variants that did not show any splice defect, RT-PCR results are shown in Fig. S5.

Six of the novel DI variants, i.e., variants c. $570+1798 \mathrm{~A}>\mathrm{G}$, c.769-788A $>\mathrm{T}, \quad$ c.859-640A $>\mathrm{G}, \quad$ c.1938-514A $>\mathrm{G}, \quad$ c.2588$706 \mathrm{C}>\mathrm{T}$, and c. $4634+741 \mathrm{~A}>\mathrm{G}$, resulted in out-of-frame $\mathrm{PE}$ inclusions in the RNA and were deemed severe (Figs. 2 and 3). Variants c.67-2023T $>\mathrm{G}$ and c.859-546G $>\mathrm{A}$ were classified to have a moderate effect as $33 \%$ and $36 \%$ of the $\mathrm{WT}$ RNA products were present, respectively. As predicted due to the presence of a downstream cryptic splice donor site (SDS), variant $c .1937+37 \mathrm{C}>\mathrm{G}$ led to an elongation of exon 13 by 36 nucleotides, which resulted in the introduction of a premature stop codon (p.Phe647*). Moreover, two intron 13 variants, c.1938-621G $>A$ and c.1938-514A $>$ G, showed a complex splice pattern that led to the generation of two mutant transcripts each (Fig. 3a-c). Each of these products contained a shared PE of 134 nt (PE1) as well as variantspecific PEs, denoted PE2 (174 nt) or PE3 (109 nt) for c.1938-621G $>$ A and c.1938-514A $>$ G, respectively (Fig. S6). For variant c.1938-621G $>A$ only $7 \%$ of the total complementary DNA (cDNA) product showed PE inclusion whereas for c.1938-514A $>$ G, 87\% of the cDNA products were mutant. To investigate the nature of the PE1 insertions, we studied the exon 12-17 segment of the mRNA obtained from photoreceptor progenitor cells (PPCs) derived from a control person. As depicted in Fig. S7, transcripts containing PE1 or PE1 and PE2 were identified when PPCs were grown under nonsense-mediated decay-suppressing conditions. The sum quantity of these two products was $2.9 \%$ of total mRNA suggesting that there are small amounts of $\mathrm{PE}$ insertions involving PE1 in the healthy retina.

Intriguingly, DI variant c.6148-84A $>\mathrm{T}$ showed four RNA splice products, namely a normal spliced RNA, the skipping of exon 45, the insertion of a 221-nt PE (pela) coupled with the deletion of exon 44, and finally, the insertion of a 173-nt PE (pelb) that consist of the same SDS as pela but a different splice acceptor site (SAS) (Fig. 3d-f). Finally, variant c.3863$1064 \mathrm{~A}>\mathrm{G}$ showed a complex splice pattern compared with the WT and variant c.6283-78G $>$ T led to the insertion of a 203-nt $\mathrm{PE}$ in intron 45 (Fig. S6). However, the exact boundaries of the presumed $\mathrm{PE}$ for variant c.3863-1064A $>\mathrm{G}$ could not yet be determined due to technical difficulties.

Overall, 13 novel DI variants were found in 18 alleles. Next to the novel variants, 14 previously reported pathogenic DI variants $^{7-9,13,18}$ were found in a total of 99 alleles, details of which are shown in Fig. $\mathbf{1 b}$ and Table S8.

\section{Identification of novel structural variants in STGD1 cases} Among 1054 STGD and STGD-like patients analyzed, we identified 11 unique novel heterozygous SVs, all exonspanning deletions, in 16 patients. The corresponding deletions encompass between 1 and 33 exons, ranging from $411 \mathrm{bp}$ to $55.7 \mathrm{~kb}$ (Fig. 4, Tables S12-S17). All deletions were found in a heterozygous state in single cases, except the smallest (c.699_768+341del), which encompassed $70 \mathrm{bp}$ of exon 6 and 341 bp of intron 6 , and was found in six unrelated patients of Spanish origin. Deletion breakpoints were determined employing genomic PCR and Sanger sequencing for 9 of the 11 deletions. Two deletion junctions (deletions 7 and 11) could not be amplified as the 3' breakpoints were located downstream of the gene beyond the regions targeted by smMIPs. Surprisingly, Sanger sequencing revealed two complex rearrangements as deletions 5 and 6 carried inverted fragments of 279 and $224 \mathrm{bp}$ respectively, residing between large deletions. These small inversions could not be identified with the CNV detection tool.

\section{Microhomologies, repetitive elements, and non-B DNA conformation at deletion breakpoints}

The breakpoints of the deletions were subjected to bioinformatic analysis to find elements underlying their formation. The presence of microhomology, repetitive elements, and non-B DNA conformations was investigated except for deletions 7 and 11 as exact boundaries could not be determined by Sanger sequencing. All other studied SVs presented microhomology at the breakpoint junctions, ranging in size from 1 to $6 \mathrm{bp}$ (Fig. S8), four of which presented short insertions (Table S18). In 8 of $11(72.7 \%)$ of the deletion breakpoints, a known repetitive element was observed, including seven non-long terminal repeats (nonLTR) retrotransposons, among which there were one short interspersed nuclear element (SINE) and four long interspersed nuclear elements (LINEs), three DNA transposons from the $h A T$ superfamily, and two retrotransposons from the LTR superfamily. However, no breakpoint was part of a known element belonging to the same class and no Alu sequence was observed at the breakpoint junctions. Finally, the most prevalent non-B conformations observed among our breakpoints are $\operatorname{Oligo}(\mathrm{G}) \mathrm{n}$ tracts as 21 of these repeats were found in seven SVs (Tables S18, S19). Inverted repeats were observed in five breakpoint regions. No direct repeats or mirror repeats have been detected, therefore excluding triplex and slipped hairpin structure formation, respectively.

\section{Uniparental isodisomy of chromosome 1}

In STGD1 proband DNA14-33085, a causal homozygous DI variant, c.859-506G >C (p.[Phe287Thrfs*32,=]), was identified. Segregation analysis revealed this variant to be present in his unaffected father, but not in his unaffected mother. To test the possibility that the mother carried a deletion 

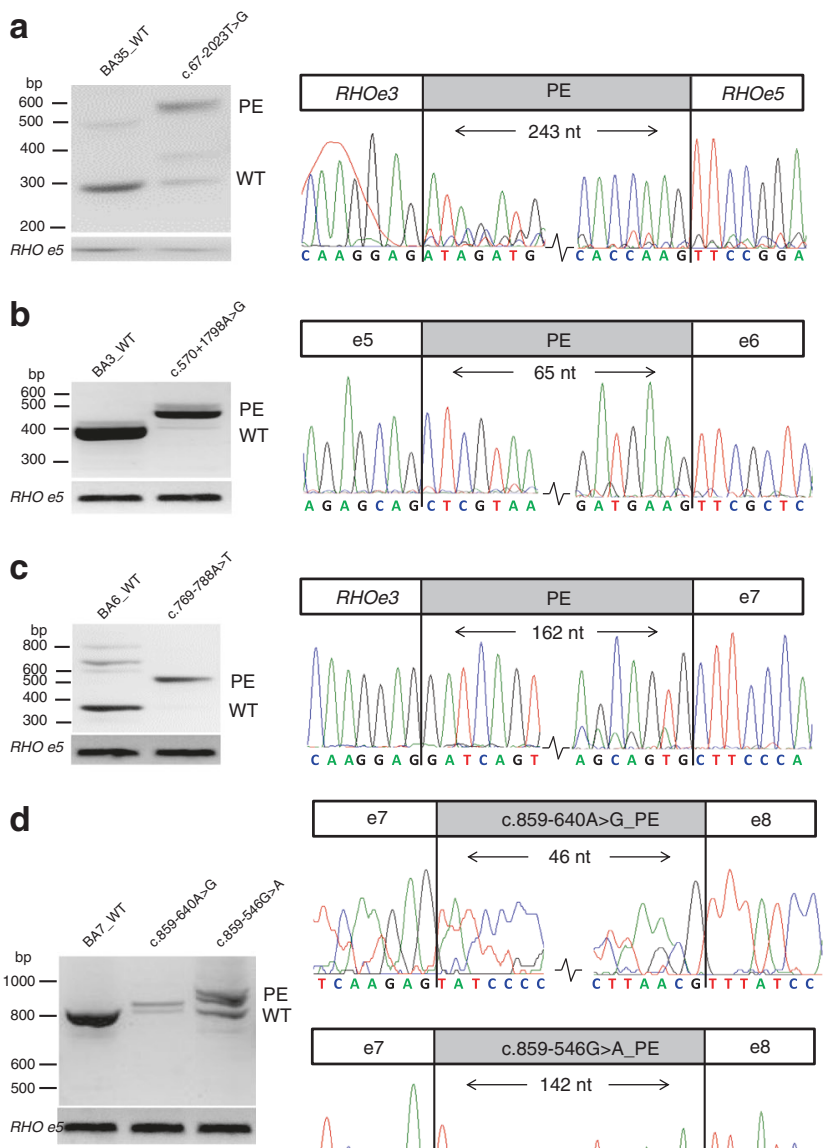

PE
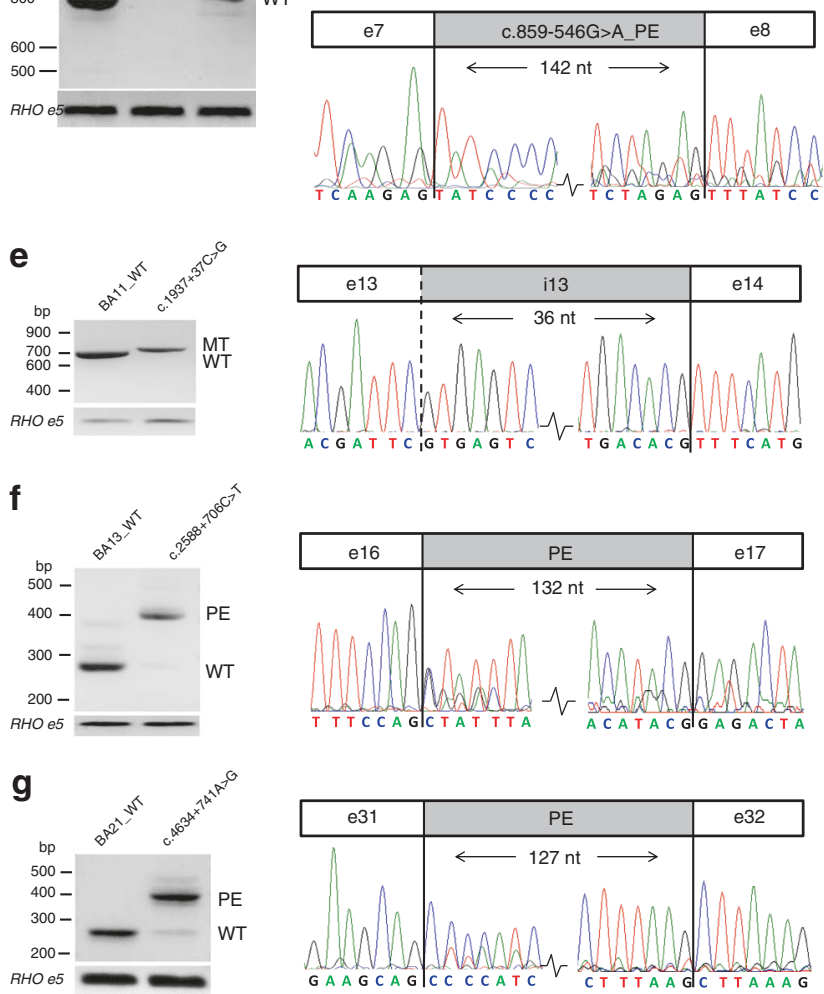

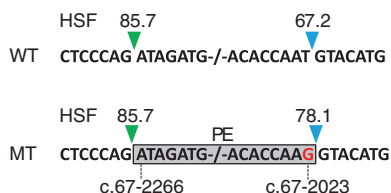

HSF 93.5

n.a.

WT TCtTCAG CtcGtAA-/-GatgaAG ATAAGGA
HSF 93.5 PE 97.1
MT tcttcaG CTCGTAA-/-GATGAAG GTAAGgA c. $570+1733 \quad$ C. $570+1797$

HSF $82.9 \quad 92.3$

WT TGACTCCTCTAG GATC-/-AGCAGTG GTGAGTT

HSF $\quad 86.1 \quad$ PE $\quad 92.3$

MT TGTCTCCTCTAG GATC-PE -AGCAGTG GTGAGTT $\begin{array}{ll}\text { c.769-778 } \quad \text { c.769-617 } & \end{array}$

$\begin{array}{ll}\text { HSF } & 93.6 \quad 69.2\end{array}$

WT TCCCCAG TATCCCC-/-CTTAACA GTAAAAT

HSF $\quad 93.6$ PE $\quad 79.8$

MT TCCCCAG TATCCCC-I-CTTAACG GTAAAAT $\begin{array}{ll}\text { c. } 859-685 \quad \text { c.859-640 } & 0\end{array}$

HSF $93.6 \quad 76.3$

WT TCCCCAG TATCCCC-I-CTCTAGGG GTCACT

HSF $93.6 \quad$ PE $\quad 81.2$

MT TCCCCAGTATCCCC-1-CTCTAGAGGTCACT

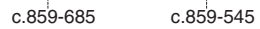

HSF $\quad 81.1 \quad$ n.a.

WT CGTGGACGATTC GTGAG-I-CG CTA

HSF Exon13 $76.6 \quad 80.0$

MT CGTGGACGATTCGTGAGG-I-CGG:GTA

$+36$

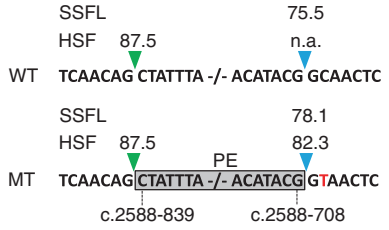

HSF 86.0 n.a.

WT tTATCAG CCCCATC-I-CTTtaAG ATAAAAC

HSF $\quad 93.6 \quad$ PE $\quad 84.5$

MT TTATCAG[CCCCATC-I-CTTIAAGGTAAAAC

$\begin{array}{ll}\text { c. } 4634+614 & \text { c. } 4634+740\end{array}$

Fig. 2 Novel splice defects due to deep-intronic $\boldsymbol{A B C A 4}$ variants. Wild-type (WT) and mutant (MT) midigenes were transfected in HEK293T cells and the extracted RNA was subjected to reverse-transcription polymerase chain reaction (RT-PCR). Left panels show the ABCA4-specific RT-PCR products with Rhodopsin exon 5 ( $R H O$ e5) RT-PCR as a transfection efficiency control. In the middle panels, Sanger sequencing results of the RT-PCR products are given. At the right side, pseudoexons (PEs) and an exon elongation are depicted with splice site strength predictions for WT and MT sequences, with green rectangles representing the splice acceptor sites and blue rectangles representing the splice donor sites. Red highlighted nucleotides represent the variants. Except for c. $1937+37$ C > G (e), which resulted in a 36-nt exon 13 elongation, all deep-intronic variants lead to PEs (a-d, $\mathbf{f}, \mathbf{g})$. The intron 7 variants in (d) result in partially overlapping PEs that share the same splice acceptor site at position c.859-685. HSF Human Splicing Finder, na not applicable, PE pseudoexon, SSFL splice site finder-like. 
a

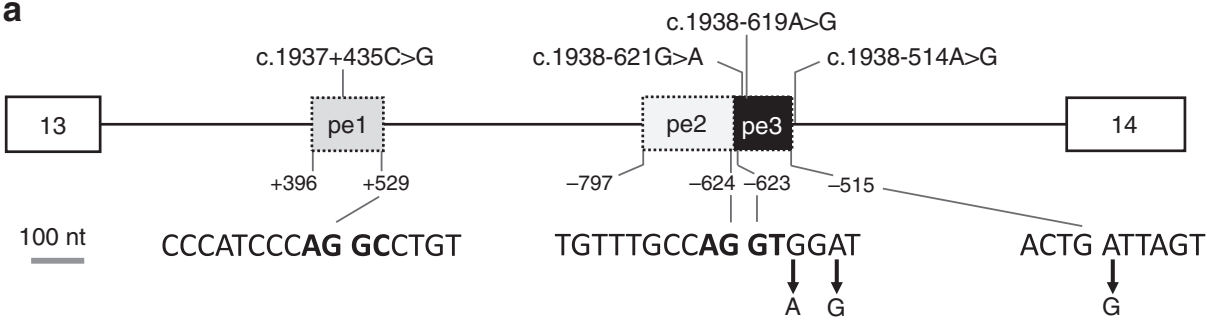

HSF $78.3 \rightarrow 79.5 \quad 90.5 \quad 90.2$

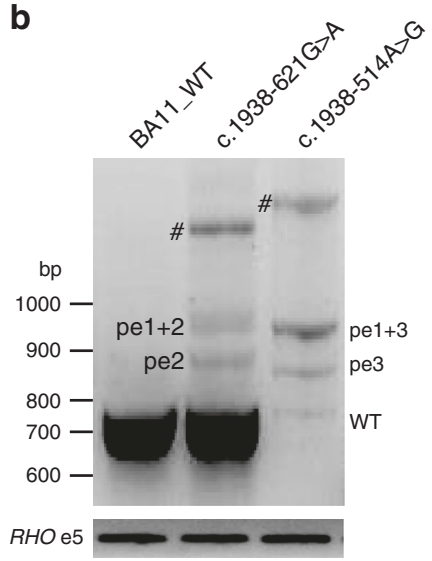

C

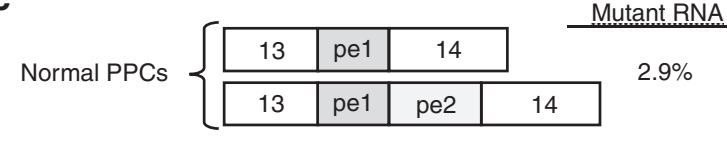

\begin{tabular}{ll|l|l|l|}
\cline { 3 - 4 }$\quad 1937+435 C>G \quad 8.9 \%$ \\
\cline { 2 - 4 }$\quad 13$ & pe1 & 14
\end{tabular}

c.1938-619A $>\mathrm{G}\left\{\begin{array}{|l|c|c|c|}\hline 13 & \text { pe2 } & \multicolumn{2}{|c|}{14} \\ \hline \hline 13 & \text { pe1 } & \text { pe2 } & 14 \\ \hline\end{array} 80.0 \%\right.$ **

c.1938-621G $>A\left\{\begin{array}{|l|c|c|c|}\hline 13 & \text { pe2 } & \multicolumn{2}{|c|}{14} \\ \hline 13 & \text { pe1 } & \text { pe2 } & 14 \\ \hline\end{array}\right.$

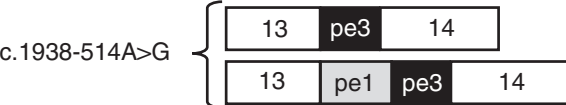

$87.0 \%$

d

c. $6148-84 \mathrm{~A}>\mathrm{T}$

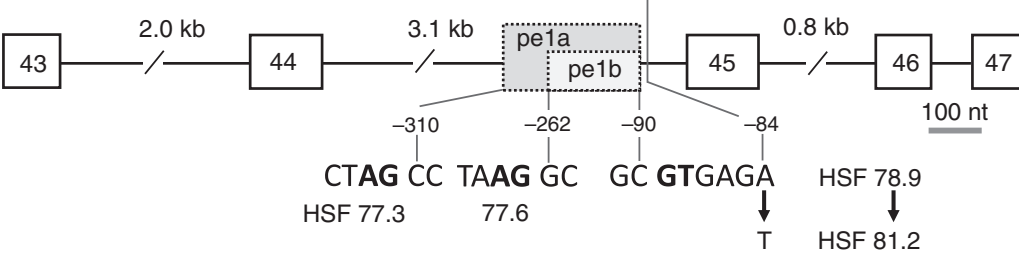

e

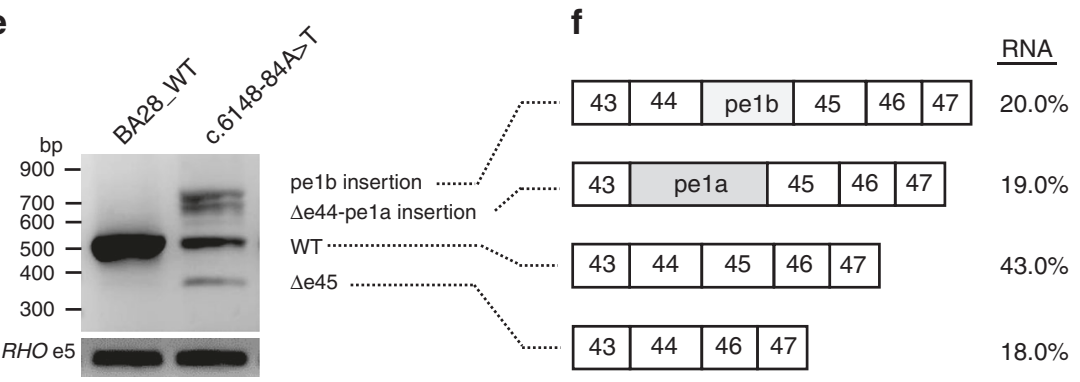

Fig. 3 Splice defects due to variants in ABCA4 introns 13 and 44. a Genomic structure of intron 13 containing three pseudoexons (PEs) due to four deep-intronic variants. PE2 and PE3 share a splice donor site (SDS) (for PE2) and splice acceptor site (for PE3). Variants C. 1938-621G>A and c.1938-619A>G strengthen the same cryptic SDS of PE2 slightly or strongly, respectively, as based on the Human Splicing Finder (HSF). Variant c. 1938-514A>G creates a new strong SDS of PE3. The canonical and putative canonical splice sequences are given in bold lettering. The first and last positions of the PEs are provided. b Agarose gel analysis of reverse-transcription polymerase chain reaction (RT-PCR) products for intron 13 variants upon HEK293T cell splice assays. PE2 and PE3 were observed as single insertions, but also in combination with PE1. "Heteroduplex fragments of the lower bands. c Schematic representation of all mutant transcripts identified upon RT-PCR in HEK293T cell splice assays and of PE1 and PE1/PE2 observed as naturally occurring PEs when analyzing photoreceptor progenitor cells (PPCs) derived from a healthy individual. Interestingly, PE1 was previously shown to be induced by variant C. 1937+435C $>$ G (*Sangermano et al.) ${ }^{9}$ and also can be part of mutant transcripts, together with PE2 or PE3. This is surprising as it is located far upstream of the other causal variants. ${ }^{*}$ Reported by Fadaie et al. ${ }^{13} \mathbf{d}$ Variant c.6148-84A>T strengthens a SDS and results in PE1a or PE1b by employing upstream or downstream splice acceptor sites, respectively. These splice acceptor sites are comparable in predicted strength based on HSF. The canonical splice sequences are given in bold. e Agarose gel analysis of RT-PCR products due to c.6148-84A>T. f The largest fragment shows a 173-nt PE insertion between exons 44 and 45 . The second largest band contains a 221-nt PE insertion (PE1a) and skipping of exon 44. The third-largest fragment represents the WT messenger RNA (mRNA) and the smallest fragment misses exon 45. The relative amounts of the products are listed at the right side. 


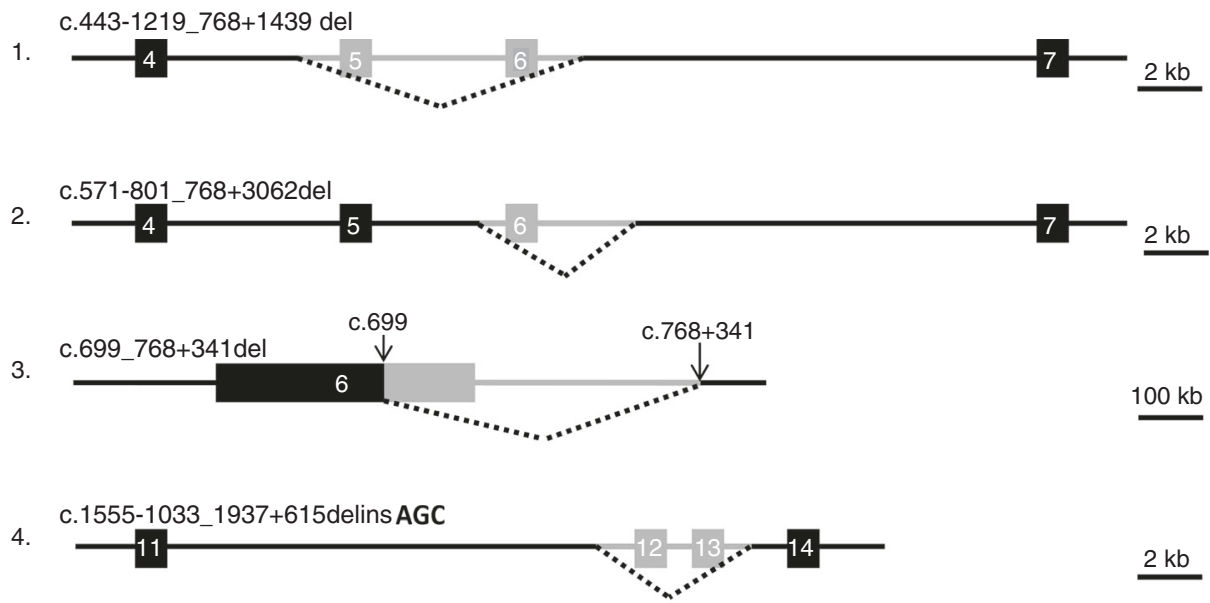

C.1555-3491_1938+83delins1734_1761-107inv

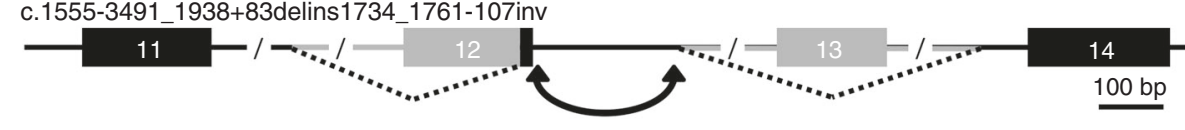

C.1555-2428_2161-101delins2160+7_2160+230invATGAATGins
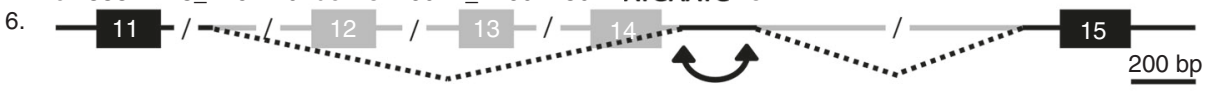

c. $\left(2653+1\right.$ 2654-1) ( ${ }^{\star} 1$ ? $)$ del

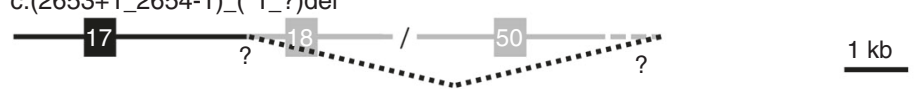

c.4254-197_4672delins GCTTTTT

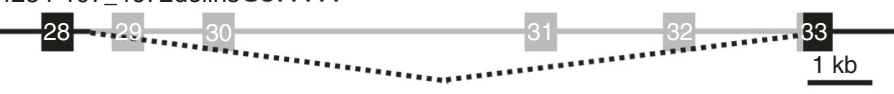

9.

c.6005+658_6147+757delinsTTTAACAGTGTT

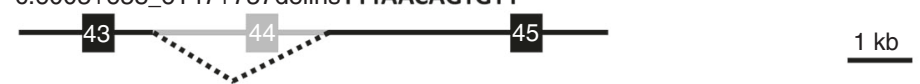

10.

c.6282+63_6546del

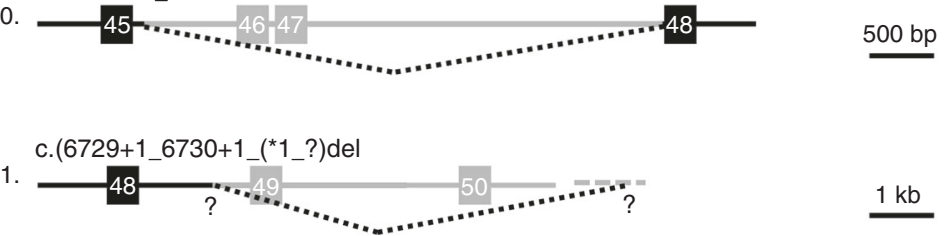

Fig. 4 Novel heterozygous structural variants in $\boldsymbol{A B C A 4}$. Schematic representation of the 11 structural variants identified. Exons are represented as boxes, black when they are not deleted and gray when they are deleted. Introns are represented as continuous lines, whereas stippled lines depict the deleted regions. Question marks denote that the exact location of breakpoints were unknown. Inverted double arrows represent inverted sequences.

spanning this variant, we performed $\mathrm{CNV}$ analysis in the proband's $A B C A 4$ gene. No deletion was identified (Table S17, column AU) and no heterozygous SNPs were observed in or near ABCA4 in the proband's DNA. To test whether the chromosome 1 of the father carrying the c.859$506 \mathrm{G}>\mathrm{C} A B C A 4$ variant was passed on to the proband as two copies (UPD), exome sequencing was conducted for the proband's DNA. As shown in Fig. S9, chromosome 1 of the proband carries only homozygous SNPs, strongly suggesting the occurrence of UPD.

\section{DISCUSSION}

Employing 3866 smMIPs, $97.4 \%$ of the $128-\mathrm{kb}$ ABCA4 gene could be sequenced robustly in 1054 genetically unsolved probands with a STGD or a STGD-like phenotype. In this way, $448(42.5 \%)$ of the probands could be genetically solved. We not only identified nine novel NCSS variants and 13 novel DI variants, but also 11 novel heterozygous SVs. The large setup of this study allowed us to provide a "landscape" overview of the different variant types underlying STGD1. As depicted in Fig. 1a, we can appreciate that DI variants 
constitute a significant cause of STGD1, i.e., $11.7 \%$ of the alleles in biallelic cases, identified in $22.5 \%$ of biallelic probands. Deletions constitute $1.8 \%$ of alleles and were found in $3.5 \%$ of biallelic cases. Seven probands carried two DI variants or one DI variant and one SV. Taken together, "dark matter" alleles were found in 113/448 (25.2\%) biallelic STGD1 probands. Together, these results strongly argue for a complete sequence analysis of the ABCA4 gene to fully appreciate its mutational landscape.

\section{Complex splice defects due to intron 13 and 44 variants} Interestingly, the two intron 13 DI variants, i.e., c.1938$621 \mathrm{G}>\mathrm{A}$ and c.1938-514G $>\mathrm{A}$, were in close vicinity of two previously described variants, c. $1937+435 C>G^{9}$ and c.1938619A $>$ G. ${ }^{10,13}$ As shown in Fig. 3a-c the PE resulting from c.1937-514A $>\mathrm{G}$ (PE3) is located adjacent to PE2 as they share a dual SAS/SDS (Fig. 3a-c). The involvement of PE1, located 491,493 , and 775 nt upstream of variants c.1937-621G $>A$, c. $1937-619 A>G$, and c.1937-514A $>$ G, respectively, is very surprising. Control PPCs also show a small percentage $(2.9 \%)$ of mRNAs containing PE1 or PE1-PE2. Interestingly, the SDS of PE1 also can be employed as a SAS, which, in theory could render this intronic SAS/SDS a target for recursive splicing. ${ }^{25}$ Together, these findings suggest that there is a "natural sensitivity" for PE1 to be recognized as a PE even if the splice defect is located far downstream. Intron 44 variant c.6148$84 \mathrm{~A}>\mathrm{T}$ interestingly resulted in three abnormal splice products involving different $\mathrm{PE}$ insertions with or without flanking exon 44 or 45 deletions. Follow-up studies employing patient-derived retinal-like cells are required to validate these complex splicing patterns.

In Table S20, we listed all published 353 DI variant alleles. ${ }^{5-13,18,26,27}$ The three most frequent are c. $4253+43 \mathrm{G}>\mathrm{A}$ $(n=100)$, c. $4539+2001 \mathrm{G}>\mathrm{A}(n=64)$, and c.5196+1137G $>\mathrm{A}$ $(n=47)$. For some DI variants, the splice defects in HEK293T cells or patient-derived PPCs are very small (c.769-784C>T, c. $1937+435 \mathrm{G}>\mathrm{C}, \quad$ c. $1937-621 \mathrm{G}>\mathrm{A})^{9,28}$ (this study) or smaller than expected (c.4539+2001G>A, c.4539 $+2028 \mathrm{C}>\mathrm{T}$ ). We hypothesize that retina-specific splice factors play roles that are largely missing (HEK293T cells) or underrepresented (PPCs) compared with the normal retina.

\section{Current state of knowledge on structural variants in ABCA4} In this study, 11 unique SVs with sizes ranging from 411 bp to $55.7 \mathrm{~kb}$ were readily identified employing an easy-to-use visual detection tool taking advantage of the high number of reads obtained from smMIPs-based sequencing. Although this tool needs further automation to increase its performance for the detection of smaller deletions or duplications, it demonstrated its efficiency for deletions as small as $411 \mathrm{bp}$. To our knowledge, 47 different SVs have been identified in STGD1 patients (Table S21), 25 of which have been published elsewhere. Forty SVs are deletions, ranging in size from $23 \mathrm{bp}$ to complete deletion of the ABCA4 gene. There are six duplications, ranging from $24 \mathrm{bp}$ to $26 \mathrm{~kb}$, two indels, and one small insertion of $24 \mathrm{bp}$. As shown in Fig. S10, these SVs are spread over the entire gene. All SVs are rare, except for a 23bp deletion affecting the splicing of exons 28 and 29 in 15 Israeli probands, as well as deletions spanning exons 20-22 and exon 6, both found in 6 probands, in Belgium/Germany/ Netherlands and from Iberic origin, respectively, suggesting founder effects.

This genomic instability could be explained by the local genomic architecture (the presence of microhomology, repetitive elements, sequences forming non-B DNA conformations, and sequence motifs), leading to genomic rearrangements by impairing the replication process. For example, a microhomology of 1-4 bp may facilitate nonhomologous end joining $(\mathrm{NHEJ})^{29}$ and longer microhomologies of between 5 and $25 \mathrm{bp}$ may favor microhomology-mediated end joining (MMEJ). ${ }^{30}$ The assessment of the local architecture of deletions identified in this study lead us to rule out the non-allelic homologous recombination (NAHR) hypothesis (as no Alu sequence or L1 at any breakpoint was observed) and to propose the NHEJ or replication slippage models as the main implicated mechanisms (Table S18, Fig. S8). Indeed, the presence of microhomologies $<5 \mathrm{bp}$ in most of the junctions, and of scars characterized by insertion of several random nucleotides, could be a signature for NHEJ. Alternatively, several examples of an impaired replication fork have been noted that supports the replicativebased repair model. Indeed, despite the absence of repetitive elements of the same class at both sides of the breakpoints, their presence may initiate the formation of secondary structures, as repetitive elements could be more difficult to replicate, leading to an increased chance of replication fork stalling or collapsing. ${ }^{31}$ Finally, Oligo $(G) n$ tracts displayed a significant overrepresentation in the breakpoint regions. Such structures can induce tetraplex formation ${ }^{32}$ and could also trigger rearrangement.

\section{Uniparental isodisomy chromosome 1}

UPD was found in one STGD1 case in this study, which represents the third STGD1 case showing UPD thus far reported. $^{33,34}$ UPD is a rare event, with an estimated occurrence of 1 in 5000 or even fewer individuals. ${ }^{35}$ UPD was also described in six other inherited retinal dystrophy patients in which chromosomes 1,2, and 6 were implicated. $^{36-41}$ We cannot exclude that there are additional UPD cases in our cohort as segregation analysis was not performed for all homozygous cases. Our finding stresses the importance of segregation analysis in the parents' DNAs as the recurrence risk for future offspring is very low in UPD families.

\section{Missing heritability}

In $174 / 1054$ (16.5\%) of probands, we identified only one (likely) causal allele. In view of the high carrier frequency of $A B C A 4$ variants in the general population, estimated to be $\sim 5 \%$, ${ }^{3,14}$ about one-third of these monoallelic cases may be explained in this way. This may even be higher as we intentionally recruited monoallelic STGD and STGD-like probands for this study. Some causal variants may have 
escaped our attention. First, we have not focused on variants affecting transcription regulation. Thus far, there is limited evidence for $A B C A 4$ variants affecting transcription, ${ }^{7}$ but the reported putative regulatory variants were not found in this study. As in silico tools (Alamut algorithms, SpliceAI) ${ }^{42}$ may not predict retina-specific splice defects, we may have missed some causal variants. Also, smMIPs-based sequencing may miss heterozygous deletions smaller than $\sim 400$ bp and will not detect insertions or inversions larger than $\sim 40 \mathrm{bp}$. In addition, more refined functional tests of coding and noncoding $A B C A 4$ variants are needed to understand the full genetic landscape of STGD1.

The major advantages of smMIPs-based $A B C A 4$ sequencing compared with genome sequencing are that it (1) is at least an order of magnitude cheaper than genome sequencing, (2) results in much smaller data storage, and (3) requires no separate informed consent regarding secondary findings. Disadvantages of smMIPs are that (1) it is restricted to one or a few genes if including introns, (2) it is more cost-effective when large series are analyzed, (3) the analysis is suitable for the detection of CNVs but not for inversions and insertions, and (4) the sequencing procedure and variant calling require a specialized setup.

In our study a significant fraction of probands carried one (likely) causal variant or c.5603A $>\mathrm{T}$ as a single allele (239; $22.7 \%)$ or no causal variant $(364 ; 34.5 \%)$. A more comprehensive smMIPs-based screening platform for these STGDlike cases would likely require the sequence analysis of an additional $\sim 80$ genes associated with inherited central vision defects.

As shown in this study, smMIPs-based analysis of the complete sequence(s) of one or a few genes implicated in clinically well-defined human diseases may allow the (re) analysis of hundreds to thousands of samples, in particular by targeting cohorts in developing countries in which low-cost analysis is crucial. A similar approach can be applied to all other frequent monogenic disorders to find missing variants in noncoding regions to provide a genetic diagnosis.

In conclusion, comprehensive sequence analysis of $A B C A 4$ in 1054 unsolved STGD and STGD-like probands, splice assays in HEK293T cells, and SV analysis resulted in the identification of "dark matter" variants in $25 \%$ of biallelic STGD1 probands. Novel complex types of splice defects were identified for intron 13 and 44 variants. Together with published causal DI variants and SVs, a detailed genomic and transcriptomic landscape of $A B C A 4$-associated STGD1 was thereby established.

\section{SUPPLEMENTARY INFORMATION}

The online version of this article (https://doi.org/10.1038/s41436020-0787-4) contains supplementary material, which is available to authorized users.

\section{ACKNOWLEDGEMENTS}

We thank Ellen Blokland, Duaa Elmelik, Emeline Gorecki, Marlie Jacobs-Camps, Charlene Piriou, Mariateresa Pizzo, and Saskia van der Velde-Visser for technical assistance. We thank Béatrice Bocquet, Dominique Bonneau, Krystyna H. Chrzanowska, Hélene Dollfus, Isabelle Drumare, Monika Heusipp, Takeshi Iwata, Beata Kocyła-Karczmarewicz, Atsushi Mizota, Nobuhisa Nao-i, Adrien Pagin, Valérie Pelletier, Rafal Ploski, Agnieszka Rafalska, Rosa Riveiro, Malgorzata Rydzanicz, Blanca Garcia Sandoval, Kei Shinoda, Francesco Testa, Kazushige Tsunoda, Shinji Ueno, and Catherine Vincent-Delorme for their cooperation and ascertaining STGD1 cases. We thank Rolph Pfundt for his assistance in exome sequencing data analysis. We are grateful to the Eichler and Shendure labs (Department of Genome Sciences, University of Washington), for assistance with the initial MIP protocol. We thank the European Reference Network (ERN)-EYE and European Retinal Disease Consortium (ERDC) networks, the Japan Eye Genetics Consortium, and the East Asian Inherited Retinal Disease Society.

This work was supported by the RetinaUK, grant number GR591 (to F.P.M.C.); a Fighting Blindness Ireland grant, grant number FB18CRE (to F.P.M.C., G.J.F.); a Horizon 2020, Marie SklodowskaCurie Innovative Training Network entitled European Training Network to Diagnose, Understand and Treat Stargardt Disease; Frequent Inherited Blinding Disorder-StarT (813490) (to E.D.B., F.P. M.C., S.B., G.J.F.); Foundation Fighting Blindness USA, grant number PPA-0517-0717-RAD (to F.P.M.C.); the Rotterdamse Stichting Blindenbelangen, the Stichting Blindenhulp, and the Stichting tot Verbetering van het Lot der Blinden (to F.P.M.C.); and by the Landelijke Stichting voor Blinden en Slechtzienden, Macula Degeneratie fonds and the Stichting Blinden-Penning that contributed through Uitzicht 2016-12 (to F.P.M.C.). This work was also supported by the Algemene Nederlandse Vereniging ter Voorkoming van Blindheid and Landelijke Stichting voor Blinden en Slechtzienden that contributed through UitZicht 2014-13, together with the Rotterdamse Stichting Blindenbelangen, Stichting Blindenhulp, and the Stichting tot Verbetering van het Lot der Blinden (to F.P.M.C.). This work was also supported by Groupement de Coopération Sanitaire Interrégional G4 qui réunit les Centres Hospitaliers Universitaires Amiens, Caen, Lille et Rouen (GCS G4) and by the Fondation Stargardt France (to C.-M.D.), Federal Ministry of Education and Research (BMBF), grant numbers 01GM0851 and 01GM1108B (to B.H.F.W.), programs SVV 260516, UNCE 204064, and PROGRES-Q26/LF1 of the Charles University (to B.K., L.D., P.L.). This work was supported by grant AZV NU20-07-00182 (to P.L., B.K. and L.D.). The work of A.D. was supported by Fighting Blindness Ireland, Health Research Board of Ireland and the Medical Research Charities Group (MRCG-201614) (to G.J.F.). This work was supported by grant AZV NU20-0700182 (to P.L., B.K., L.D.). This work was also supported by the Ghent University Research Fund (BOF15/GOA/011), by the Research Foundation Flanders (FVO) G0C6715N, by the Hercules foundation AUGE/13/023 and JED Foundation (to E.D.B.). M.B. was PhD fellow of the FWO and recipient of a grant of the funds for Research in Ophthalmology (FRO). E.D.B. is Senior Clinical Investigator of the FWO (1802215N; 1802220N). The work of M. D.P-.V. is supported by the Conchita Rábago Foundation and the Boehringer Ingelheim Fonds. The work of C.A. is supported by grants PI16/0425 from ISCIII partially supported by the European 
Regional Development Fund (ERDF), RAREGenomics-CM (CAM, B2017/BMD-3721), ONCE, and Ramon Areces Foundation. This work was supported by the Peace for Sight grant (to D.S., A.A.). The work of L.R. and R.R. was supported by Retina South Africa and the South African Medical Research Council (MRC). This work was also supported by the Foundation Fighting Blindness, grant/ award number BR-GE-0214-0639-TECH and BRGE-0518-0734TECH (to T.B.-Y., D.S., H.N.); the Israeli Ministry of Health, grant/ award number 3-12583Q4 (to T.B.-Y., D.S., H.N.); Olive Young Fund, University Hospital Foundation, Edmonton (to I.M.M.); the National Science Center (Poland) grant number N N402 591640 (5916/B/P01/2011/40) (to M.O.); and UMO-2015/19/D/NZ2/03193 (to A.M.T.). This work was supported by the Italian Fondazione Roma (to S.B., F.S.), the Italian Telethon Foundation (to S.B.), and the Ministero dell'Istruzione del I'Università e della Ricerca (MIUR) under PRIN 2015 (to S.B., F.S.). M.B.G. and A.M. were supported by the Daljit $S$. and Elaine Sarkaria Charitable Foundation. The funding organizations had no role in the design or conduct of this research, and provided unrestricted grants.

\section{DISCLOSURE}

The authors declare no conflicts of interest.

Publisher's note Springer Nature remains neutral with regard to jurisdictional claims in published maps and institutional affiliations.

\section{REFERENCES}

1. Carss KJ, Arno G, Erwood M, et al. Comprehensive rare variant analysis via whole-genome sequencing to determine the molecular pathology of inherited retinal disease. Am J Hum Genet. 2017;100:75-90.

2. Blacharski PA, Newsome DA. Bilateral macular holes after Nd:YAG laser posterior capsulotomy. Am J Ophthalmol. 1988;105:417-418.

3. Cornelis SS, Bax NM, Zernant J, et al. In silico functional meta-analysis of 5,962 ABCA4 variants in 3,928 retinal dystrophy cases. Hum Mutat. 2017:38:400-408.

4. Sangermano R, Khan M, Cornelis SS, et al. ABCA4 midigenes reveal the full splice spectrum of all reported noncanonical splice site variants in Stargardt disease. Genome Res. 2018;28:100-110.

5. Khan M, Cornelis SS, Khan MI, et al. Cost-effective molecular inversion probe-based ABCA4 sequencing reveals deep-intronic variants in Stargardt disease. Hum Mutat. 2019;40:1749-1759.

6. Schulz HL, Grassmann F, Kellner $U$, et al. Mutation spectrum of the ABCA4 gene in 335 Stargardt disease patients from a multicenter German cohort-impact of selected deep intronic variants and common SNPs. Invest Ophthalmol Vis Sci. 2017;58:394-403.

7. Bauwens M, Garanto A, Sangermano R, et al. ABCA4-associated disease as a model for missing heritability in autosomal recessive disorders: novel noncoding splice, cis-regulatory, structural, and recurrent hypomorphic variants. Genet Med. 2019;21:1761-1771.

8. Braun TA, Mullins RF, Wagner AH, et al. Non-exomic and synonymous variants in ABCA4 are an important cause of Stargardt disease. Hum Mol Genet. 2013;22:5136-5145.

9. Sangermano R, Garanto A, Khan M, et al. Deep-intronic ABCA4 variants explain missing heritability in Stargardt disease and allow correction of splice defects by antisense oligonucleotides. Genet Med. 2019;21:1751-1760.

10. Zernant J, Xie YA, Ayuso C, et al. Analysis of the ABCA4 genomic locus in Stargardt disease. Hum Mol Genet. 2014;23:6797-6806.

11. Bauwens M, De Zaeytijd J, Weisschuh $N$, et al. An augmented ABCA4 screen targeting noncoding regions reveals a deep intronic founder variant in Belgian Stargardt patients. Hum Mutat. 2015;36:39-42.

12. Bax NM, Sangermano R, Roosing $S$, et al. Heterozygous deep-intronic variants and deletions in $A B C A 4$ in persons with retinal dystrophies and one exonic ABCA4 variant. Hum Mutat. 2015;36:43-47.
13. Fadaie $Z$, Khan M, Del Pozo-Valero $M$, et al. Identification of splice defects due to noncanonical splice site or deep-intronic variants in ABCA4. Hum Mutat. 2019;40:2365-2376.

14. Maugeri A, van Driel MA, van de Pol DJR, et al. The $2588 G \rightarrow C$ mutation in the $A B C R$ gene is a mild frequent founder mutation in the western European population and allows the classification of $A B C R$ mutations in patients with Stargardt disease. Am J Hum Genet. 1999:64:1024-1035.

15. Rivera $A$, White $K$, Stohr $H$, et al. A comprehensive survey of sequence variation in the $A B C A 4(A B C R)$ gene in Stargardt disease and age-related macular degeneration. Am J Hum Genet. 2000;67:800-813.

16. Jaakson K, Zernant J, Kulm M, et al. Genotyping microarray (gene chip) for the ABCR (ABCA4) gene. Hum Mutat. 2003;22:395-403.

17. Maia-Lopes S, Aguirre-Lamban J, Castelo-Branco M, Riveiro-Alvarez R, Ayuso C, Silva ED. ABCA4 mutations in Portuguese Stargardt patients: identification of new mutations and their phenotypic analysis. Mol Vis. 2009;15:584-591.

18. Albert S, Garanto A, Sangermano R, et al. Identification and rescue of splice defects caused by two neighboring deep-intronic ABCA4 mutations underlying Stargardt disease. Am J Hum Genet. 2018;102:517-527.

19. Charbel Issa P, Barnard AR, Herrmann P, Washington I, MacLaren RE. Rescue of the Stargardt phenotype in Abca4 knockout mice through inhibition of vitamin A dimerization. Proc Natl Acad Sci U S A. 2015;112:8415-8420.

20. Allocca M, Doria M, Petrillo M, et al. Serotype-dependent packaging of large genes in adeno-associated viral vectors results in effective gene delivery in mice. J Clin Invest. 2008;118:1955-1964.

21. Lu B, Malcuit C, Wang S, et al. Long-term safety and function of RPE from human embryonic stem cells in preclinical models of macular degeneration. Stem Cells. 2009;27:2126-2135.

22. Cremers FPM, Cornelis SS, Runhart EH, Astuti GDN. Author response: penetrance of the ABCA4 p.Asn1868lle allele in Stargardt disease. Invest Ophthalmol Vis Sci. 2018;59:5566-5568.

23. Runhart EH, Sangermano R, Cornelis SS, et al. The common ABCA4 variant p.Asn1868lle shows nonpenetrance and variable expression of Stargardt disease when present in trans with severe variants. Invest Ophthalmol Vis Sci. 2018;59:3220-3231.

24. Zernant J, Lee W, Collison FT, et al. Frequent hypomorphic alleles account for a significant fraction of ABCA4 disease and distinguish it from agerelated macular degeneration. J Med Genet. 2017;54:404-412.

25. Hafez M, Hausner G. Convergent evolution of twintron-like configurations: one is never enough. RNA Biol. 2015;12:1275-1288.

26. Zernant J, Lee W, Nagasaki T, et al. Extremely hypomorphic and severe deep intronic variants in the ABCA4 locus result in varying Stargardt disease phenotypes. Cold Spring Harb Mol Case Stud. 2018;4:a002733.

27. Nassisi M, Mohand-Said S, Andrieu C, et al. Prevalence of ABCA4 deepintronic variants and related phenotype in an unsolved "one-hit" cohort with Stargardt disease. Int J Mol Sci. 2019;20:5053.

28. Runhart $\mathrm{EH}$, Valkenburg D, Cornelis SS, et al. Late-onset Stargardt disease due to mild, deep-intronic ABCA4 alleles. Invest Ophthalmol Vis Sci. 2019;60:4249-4256.

29. Lieber MR. The mechanism of double-strand DNA break repair by the nonhomologous DNA end-joining pathway. Annu Rev Biochem. 2010; 79:181-211.

30. McVey M, Lee SE. MMEJ repair of double-strand breaks (director's cut): deleted sequences and alternative endings. Trends Genet. 2008;24 529-538.

31. Vissers LE, Bhatt SS, Janssen IM, et al. Rare pathogenic microdeletions and tandem duplications are microhomology-mediated and stimulated by local genomic architecture. Hum Mol Genet. 2009;18:3579-3593.

32. Bacolla A, Wells RD. Non-B DNA conformations, genomic rearrangements, and human disease. J Biol Chem. 2004;279:47411-47414.

33. Fingert JH, Eliason DA, Phillips NC, Lotery AJ, Sheffield VC, Stone EM. Case of Stargardt disease caused by uniparental isodisomy. Arch Ophthalmol. 2006;124:744-745.

34. Riveiro-Alvarez R, Valverde D, Lorda-Sanchez I, et al. Partial paternal uniparental disomy (UPD) of chromosome 1 in a patient with Stargardt disease. Mol Vis. 2007;13:96-101.

35. Liehr T. Cytogenetic contribution to uniparental disomy (UPD). Mol Cytogenet. 2010;3:8

36. Rivolta C, Berson EL, Dryja TP. Paternal uniparental heterodisomy with partial isodisomy of chromosome 1 in a patient with retinitis pigmentosa without hearing loss and a missense mutation in the Usher syndrome type II gene USH2A. Arch Ophthalmol. 2002;120:1566-1571. 
37. Roosing $\mathrm{S}$, van den Born $\mathrm{LI}$, Hoyng $\mathrm{CB}$, et al. Maternal uniparental isodisomy of chromosome 6 reveals a TULP1 mutation as a novel cause of cone dysfunction. Ophthalmology. 2013;120: 1239-1246.

38. Thompson DA, Gyurus P, Fleischer LL, et al. Genetics and phenotypes of RPE65 mutations in inherited retinal degeneration. Invest Ophthalmol Vis Sci. 2000;41:4293-4299.

39. Thompson DA, McHenry $C L$, Li Y, et al. Retinal dystrophy due to paternal isodisomy for chromosome 1 or chromosome 2, with homoallelism for mutations in RPE65 or MERTK, respectively. Am J Hum Genet. 2002 70:224-229.
40. Wiszniewski W, Lewis RA, Lupski JR. Achromatopsia: the CNGB3 p. T383fsX mutation results from a founder effect and is responsible for the visual phenotype in the original report of uniparental disomy 14 . Hum Genet. 2007;121:433-439.

41. Souzeau E, Thompson JA, McLaren TL, et al. Maternal uniparental isodisomy of chromosome 6 unmasks a novel variant in TULP1 in a patient with early onset retinal dystrophy. Mol Vis. 2018;24:478-484

42. Jaganathan K, Kyriazopoulou Panagiotopoulou S, McRae JF, et al. Predicting splicing from primary sequence with deep learning. Cell. 2019;176:535-48 e524.

Mubeen Khan, MSc $\mathrm{M}^{1,2}$, Stéphanie S. Cornelis, MSc ${ }^{1,2}$, Marta Del Pozo-Valero, MSc, Laura Whelan, $\mathrm{BSc}^{4}$, Esmee H. Runhart, $\mathrm{MD}^{2,5}$, Ketan Mishra, MSc ${ }^{1,2}$, Femke Bults, $\mathrm{BSc}^{1}$, Yahya AlSwaiti, MD ${ }^{6}$, Alaa AlTalbishi, MD, PhD ${ }^{6}$, Elfride De Baere, MD, PhD ${ }^{7}$, Sandro Banfi, $\mathrm{MD}^{8}$, Eyal Banin, MD, $\mathrm{PhD}^{9}$, Miriam Bauwens, $\mathrm{PhD}^{7}$, Tamar Ben-Yosef, $\mathrm{PhD}^{10}$, Camiel J. F. Boon, MD, PhD ${ }^{11,12}$, L. Ingeborgh van den Born, MD, PhD ${ }^{13,14}$, Sabine Defoort, MD ${ }^{15}$, Aurore Devos, $\mathrm{MSc}^{16}$, Adrian Dockery, $\mathrm{PhD}^{4}$, Lubica Dudakova, $\mathrm{PhD}^{17}$, Ana Fakin, MD, $\mathrm{PhD}^{18}$ G. Jane Farrar, PhD ${ }^{4}$, Juliana Maria Ferraz Sallum, MD, PhD ${ }^{19,20}$, Kaoru Fujinami, MD, PhD ${ }^{21,22,23,24}$, Christian Gilissen, PhD ${ }^{1,25}$, Damjan Glavač, PhD ${ }^{26}$, Michael B. Gorin, MD, PhD ${ }^{27,28}$, Jacquie Greenberg, $\mathrm{PhD}^{29}$, Takaaki Hayashi, MD, PhD ${ }^{30}$, Ymkje M. Hettinga, MD ${ }^{31}$, Alexander Hoischen, $\mathrm{PhD}^{1}$, Carel B. Hoyng, MD, PhD ${ }^{2,5}$, Karsten Hufendiek, $\mathrm{MD}^{32}$, Herbert Jägle, $\mathrm{MD}^{33}$, Smaragda Kamakari, $\mathrm{PhD}^{34}$, Marianthi Karali, $\mathrm{PhD}^{8}$, Ulrich Kellner, $\mathrm{MD}^{35,36}$, Caroline C. W. Klaver, MD, PhD 5,37,38, Bohdan Kousal, MD7,39, Tina M. Lamey, PhD ${ }^{40,41}$, lan M. MacDonald, MD, CM ${ }^{42}$, Anna Matynia, PhD 27,28 , Terri L. McLaren, $\mathrm{BSc}^{40,41}$, Marcela D. Mena, $\mathrm{PhD}^{43}$, Isabelle Meunier, MD, PhD ${ }^{44}$, Rianne Miller, $\mathrm{BSC}^{1}$, Hadas Newman, MD ${ }^{45,46}$, Buhle Ntozini, $\mathrm{BSc}^{29}$, Monika Oldak, MD, PhD ${ }^{47}$, Marc Pieterse, MSc ${ }^{1}$, Osvaldo L. Podhajcer, $\mathrm{PhD}^{43}$, Bernard Puech, MD ${ }^{15}$, Raj Ramesar, MBA, PhD ${ }^{29}$, Klaus Rüther, MD, PhD ${ }^{48}$, Manar Salameh, BSc $^{6}$, Mariana Vallim Salles, MD ${ }^{19,20}$, Dror Sharon, $\mathrm{PhD}^{9}$, Francesca Simonelli, MD, PhD ${ }^{49}$, Georg Spital, MD ${ }^{50}$, Marloes Steehouwer, BSc ${ }^{1}$, Jacek P. Szaflik, MD, PhD ${ }^{51}$, Jennifer A. Thompson, $\mathrm{PhD}^{41}$, Caroline Thuillier, MSc ${ }^{52}$, Anna M. Tracewska, PhD ${ }^{53}$, Martine van Zweeden, $\mathrm{BSc}^{1}$, Andrea L. Vincent, MBChB, MD ${ }^{54,55}$, Xavier Zanlonghi, MD, $\mathrm{PhD}^{56}$, Petra Liskova, MD, PhD ${ }^{17,39}$, Heidi Stöhr, PhD ${ }^{57}$, John N. De Roach, $\mathrm{PhD}^{40,41}$, Carmen Ayuso, MD, PhD ${ }^{3}$, Lisa Roberts, PhD ${ }^{29}$, Bernhard H. F. Weber, $\mathrm{PhD}^{57}$, Claire-Marie Dhaenens, MD, PhD ${ }^{1,16}$ and Frans P. M. Cremers, PhD $\oplus^{1,2}$

${ }^{1}$ Department of Human Genetics, Radboud University Medical Center, Nijmegen, The Netherlands; ${ }^{2}$ Donders Institute for Brain, Cognition and Behaviour, Radboud University Medical Center, Nijmegen, The Netherlands; ${ }^{3}$ Department of Genetics, IIS-Fundación Jiménez Díaz, CIBERER, Madrid, Spain; ${ }^{4}$ The School of Genetics \& Microbiology, Trinity College Dublin, Dublin, Ireland;

${ }^{5}$ Department of Ophthalmology, Radboud University Medical Center, Nijmegen, The Netherlands; ${ }^{6}$ St John of Jerusalem Eye Hospital Group, East Jerusalem, Palestine; ${ }^{7}$ Center for Medical Genetics Ghent, Ghent University and Ghent University Hospital, Ghent, Belgium; ${ }^{8}$ Department of Precision Medicine, University of Campania Luigi Vanvitelli, Naples and Telethon Institute of Genetics and Medicine (TIGEM), Pozzuoli, Italy; ${ }^{9}$ Department of Ophthalmology, Hadassah Medical Center, Faculty of Medicine, The Hebrew University of Jerusalem, Jerusalem, Israel; ${ }^{10}$ Ruth and Bruce Rappaport Faculty of Medicine, Technion-Israel Institute of Technology, Haifa, Israel; ${ }^{11}$ Department of Ophthalmology, Leiden University Medical Center, Leiden, The Netherlands; ${ }^{12}$ Department of Ophthalmology, Amsterdam University Medical Centers, Amsterdam, The Netherlands; ${ }^{13}$ The Rotterdam Eye Hospital, Rotterdam, The Netherlands; ${ }^{14}$ The Rotterdam Ophthalmic Institute, Rotterdam, The Netherlands; ${ }^{15}$ Service d'exploration de la vision et neuro-ophtalmologie, Centre Hospitalier Universitaire de Lille, Lille, France; ${ }^{16}$ Univ. Lille, Inserm, CHU Lille, U1172 LilNCog - Lille Neuroscience \& Cognition, F-59000 Lille, France; ${ }^{17}$ Research Unit for Rare Diseases, Department of Paediatrics and Adolescent Medicine, First Faculty of Medicine, Charles University and General University Hospital in Prague, Prague, Czech Republic; ${ }^{18}$ Eye Hospital, University Medical Centre Ljubljana, Ljubljana, Slovenia; ${ }^{19}$ Department of Ophthalmology and Visual Sciences, Universidade Federal de São Paulo, São Paulo, SP, Brazil; ${ }^{20}$ Instituto de Genética Ocular, São Paulo, SP, Brazil; ${ }^{21}$ UCL Institute of Ophthalmology, London, UK; ${ }^{22}$ Laboratory of Visual Physiology, Division of Vision Research, National Institute of Sensory Organs, National Hospital Organization Tokyo Medical Center, Tokyo, Japan; ${ }^{23}$ Graduate School of Health Management, Keio University, Tokyo, Japan; ${ }^{24}$ Moorfields Eye Hospital, London, UK; ${ }^{25}$ Radboud Institute for Molecular Life Sciences, Radboud University Medical Center, Nijmegen, The Netherlands; ${ }^{26}$ Department of Molecular Genetics, Institute of Pathology, University of 
Ljubljana, Ljubljana, Slovenia; ${ }^{27}$ Department of Ophthalmology, David Geffen School of Medicine, Stein Eye Institute, University of California-Los Angeles, Los Angeles, CA, USA; ${ }^{28}$ Department of Human Genetics, David Geffen School of Medicine, University of California-Los Angeles, Los Angeles, CA, USA; ${ }^{29}$ University of Cape Town/MRC Genomic and Precision Medicine Research Unit, Division of Human Genetics, Department of Pathology, Institute of Infectious Disease and Molecular Medicine (IDM), Faculty of Health Sciences, University of Cape Town, Cape Town, South Africa; ${ }^{30}$ Department of Ophthalmology, The Jikei University School of Medicine, Tokyo, Japan; ${ }^{31}$ Bartiméus Diagnostic Center for Complex Visual Disorders, Zeist, The Netherlands; ${ }^{32}$ University Eye Hospital Hannover Medical School, Hannover, Germany; ${ }^{33}$ Department of Ophthalmology, University Hospital, University Regensburg, Regensburg, Germany; ${ }^{34}$ Ophthalmic Genetics Unit, OMMA Ophthalmological Institute of Athens, Athens, Greece; ${ }^{35}$ Rare Retinal Disease Center, AugenZentrum Siegburg, MVZ ADTC Siegburg GmbH, Siegburg, Germany; ${ }^{36}$ RetinaScience, Bonn, Germany; ${ }^{37}$ Department of Ophthalmology, Erasmus Medical Centre, Rotterdam, The Netherlands; ${ }^{38}$ Department of Epidemiology, Erasmus Medical Centre, Rotterdam, The Netherlands; ${ }^{39}$ Department of Ophthalmology, First Faculty of Medicine, Charles University and General University Hospital in Prague, Prague, Czech Republic; ${ }^{40}$ Centre for Ophthalmology and Visual Science, The University of Western Australia, Nedlands, WA, Australia; ${ }^{41}$ Australian Inherited Retinal Disease Registry and DNA Bank, Department of Medical Technology and Physics, Sir Charles Gairdner Hospital, Nedlands, WA, Australia; ${ }^{42}$ Departments of Ophthalmology and Medical Genetics, University of Alberta, Edmonton, AB, Canada; ${ }^{43}$ Laboratory of Molecular and Cellular Therapy, Fundacion Instituto Leloir-CONICET, Buenos Aires, Argentina; ${ }^{44}$ Institut des Neurosciences de Montpellier, INSERM, Université de Montpellier, Montpellier, France; ${ }^{45}$ Department of Ophthalmology, Tel Aviv Sourasky Medical Center, Tel Aviv, Israel; ${ }^{46}$ Sackler Faculty of Medicine, Tel Aviv University, Tel Aviv, Israel; ${ }^{47}$ Department of Histology and Embryology, Medical University of Warsaw, Warsaw, Poland; ${ }^{48}$ Augenarztpraxis, Dorotheenstraße, Berlin, Germany; ${ }^{49}$ Eye Clinic, Multidisciplinary Department of Medical, Surgical and Dental Sciences, University of Campania Luigi Vanvitelli, Naples, Italy; ${ }^{50}$ Department of Ophthalmology, St. Franziskus-Hospital, Münster, Germany; ${ }^{51}$ Department of Ophthalmology, Medical University of Warsaw SPKSO Ophthalmic University Hospital, Warsaw, Poland; ${ }^{52} \mathrm{CHU}$ Lille, Institut de Génétique Médicale, Lille, France; ${ }^{53}$ DNA Analysis Unit, ŁUKASIEWICZ Research Network-PORT Polish Center for Technology Development, Wroclaw, Poland; ${ }^{54}$ Department of Ophthalmology, New Zealand National Eye Centre, Faculty of Medical and Health Sciences, The University of Auckland, Grafton, Auckland, New Zealand; ${ }^{55}$ Eye Department, Greenlane Clinical Centre, Auckland District Health Board, Auckland, New Zealand; ${ }^{56}$ Centre de Compétence Maladie Rare, Clinique Jules Verne, Nantes, France; ${ }^{57}$ Institute of Human Genetics, University of Regensburg, Regensburg, Germany. 